\title{
Sharp bounds for Hardy type operators on higher-dimensional product spaces
}

\author{
Shanzhen Lu', Dunyan Yan² and Fayou Zhao ${ }^{3 *}$
}

"Correspondence:

fyzhao@shu.edu.cn

${ }^{3}$ Department of Mathematics,

Shanghai University, Shanghai,

200444, P.R. China

Full list of author information is

available at the end of the article

\begin{abstract}
A new class of Hardy type operator defined on a higher-dimensional product space is discussed. It includes two different kinds of the classical Hardy operators. In addition, we also consider the fractional Hardy operator $\mathbb{H}_{\beta}$. The bound of operator $\mathbb{H}_{\beta}$ from $L^{p}$ to $L^{a}$ is explicitly worked out. Especially, the bound of operator $\mathbb{H}_{\beta}$ from $L^{1}$ to $L^{\frac{n}{n-\beta}}, \infty$ is sharp.

MSC: 26D10; 26D15; 42B99
\end{abstract}

Keywords: sharp bound; product space; the fractional Hardy operator

\section{Introduction}

The most fundamental averaging operator is the Hardy operator defined by

$$
H(f)(x)=\frac{1}{x} \int_{0}^{x} f(t) d t
$$

where the function $f$ is a nonnegative integrable on $\mathbb{R}_{+}=(0, \infty)$ and $x>0$. A classical inequality, due to Hardy [1], states that

$$
\|H(f)\|_{L^{p}} \leq \frac{p}{p-1}\|f\|_{L^{p}}
$$

holds for $1<p<\infty$, and the constant $\frac{p}{p-1}$ is best possible.

For the multidimensional case $n \geq 2$, generally speaking, there exist two different definitions. One is the rectangle averaging operator defined by

$$
\mathfrak{R}_{n}(f)\left(x_{1}, \ldots, x_{n}\right)=\frac{1}{x_{1} \cdots x_{n}} \int_{0}^{x_{1}} \cdots \int_{0}^{x_{n}} f\left(t_{1}, \ldots, t_{n}\right) d t_{1} \cdots d t_{n},
$$

where the function $f$ is a nonnegative measurable function on $G=(0, \infty)^{n}$ and $x_{i}>0$, $i=1,2, \ldots, n$.

Another definition is the spherical averaging operator, which was given by Christ and Grafakos in [2] as follows:

$$
\mathcal{H}(f)(x)=\frac{1}{|B(0,|x|)|} \int_{|y|<|x|} f(y) d y, \quad x \in \mathbb{R}^{n} \backslash\{0\},
$$

where $f$ is a nonnegative measurable function on $\mathbb{R}^{n}$.

\section{Springer}

(0) 2013 Lu et al.; licensee Springer. This is an Open Access article distributed under the terms of the Creative Commons Attribution License (http://creativecommons.org/licenses/by/2.0), which permits unrestricted use, distribution, and reproduction in any medium, provided the original work is properly cited. 
The boundedness of operator $\mathfrak{R}_{n}$ are discussed in many papers (cf. [3-6]). $\left\|\Re_{n}\right\|_{L^{p} \rightarrow L^{p}}$, the norm of $\Re_{n}$, is $\left(\frac{p}{p-1}\right)^{n}$ and obviously depends on the dimension of the space. However, $\|\mathcal{H}\|_{L^{p} \rightarrow L^{p}}$, the norm of $\mathcal{H}$, is still $\frac{p}{p-1}$, and does not depend on the dimension of the space. The reason of generating the difference of the two kind of operators is, roughly speaking, that each variable can independently dilate by itself in the operator $\mathfrak{R}_{n}$, nevertheless, for the operator $\mathcal{H}$, all variables dilate by the same scale simultaneously. Generally speaking, the spherical averaging operator has better properties than the rectangle averaging operator does, such as the Hardy-Littlewood maximal function. A detailed account of the history of the topic can be found in the book [7]; see also Kufner and Persson's book [8].

For the operator $\mathfrak{R}_{n}$, we note that every variable is defined on the one-dimensional space. In this paper, we shall extent the definition of $\mathfrak{R}_{n}$ so that every variable is spherical average defined on a higher-dimensional space.

Next, we will give the definition of Hardy type operator on higher-dimensional product spaces as follows and discuss the corresponding properties.

Definition 1.1 Let $m \in \mathbb{N}, n_{i} \in \mathbb{N}, x_{i} \in \mathbb{R}^{n_{i}}, 1 \leq i \leq m$, and $f$ be a nonnegative measurable function on $\mathbb{R}^{n_{1}} \times \mathbb{R}^{n_{2}} \times \cdots \times \mathbb{R}^{n_{m}}$. The Hardy type operator is defined by

$$
\mathcal{H}_{m}(f)(x)=\left(\prod_{i=1}^{m} \frac{1}{\left|B\left(0,\left|x_{i}\right|\right)\right|}\right) \int_{\left|y_{1}\right|<\left|x_{1}\right|} \cdots \int_{\left|y_{m}\right|<\left|x_{m}\right|} f\left(y_{1}, \ldots, y_{m}\right) d y_{1} \cdots d y_{m},
$$

where $x=\left(x_{1}, x_{2}, \ldots, x_{m}\right) \in \mathbb{R}^{n_{1}} \times \mathbb{R}^{n_{2}} \times \cdots \times \mathbb{R}^{n_{m}}$ with $\prod_{i=1}^{m}\left|x_{i}\right| \neq 0$.

Our first aim in this paper is to provide transparent treatments of multivariate inequalities of higher-dimensional Hardy type both for the rectangle and for the ball case. Our results subsume those of [9] and [6]. In fact, if $m=1$, then the operator $\mathcal{H}_{m}$ will become $\mathcal{H}$ defined by (2); if $n_{1}=n_{2}=\cdots=n_{m}=1$, then $\mathcal{H}_{m}$ will become $\mathfrak{R}_{n}$ defined by (1). Consequently, the operator $\mathcal{H}_{m}$ includes both $\mathfrak{R}_{n}$ and $\mathcal{H}$. It is much significant to discuss the properties of $\mathcal{H}_{m}$.

Our second aim is to consider the fractional Hardy operator on the Lebesgue spaces. Recall that, for a nonnegative measurable function $f$ on $\mathbb{R}^{n}$, the $n$-dimensional fractional Hardy operator $\mathbb{H}_{\beta}$ with spherical mean is defined by

$$
\mathbb{H}_{\beta}(f)(x)=\frac{1}{|B(0,|x|)|^{1-\frac{\beta}{n}}} \int_{|y|<|x|} f(y) d y, \quad x \in \mathbb{R}^{n} \backslash\{0\},
$$

where $0 \leq \beta<n$ (cf. [10]). Clearly, $\mathbb{H}_{\beta}(|f|)(x) \leq C M_{\beta}(f)(x)$, where $M_{\beta}$ is the fractional Hardy-Littlewood maximal function defined by

$$
M_{\beta}(f)(x)=\sup _{r>0} \frac{1}{|B(x, r)|^{1-\frac{\beta}{n}}} \int_{|y-x|<r}|f(y)| d y,
$$

where $f$ is a measurable function.

For $0 \leq \beta<n, 1<p \leq \frac{n}{\beta}$, and $\frac{1}{p}-\frac{1}{q}=\frac{\beta}{n}$, the following two statements (a) and (b) are well-known (cf. [11]). 
(a) If $f \in L^{p}\left(\mathbb{R}^{n}\right)$, then

$$
\left\|M_{\beta}(f)\right\|_{L^{q}} \leq C\|f\|_{L^{p}}
$$

(b) if $f \in L^{1}\left(\mathbb{R}^{n}\right)$, then, for any $\lambda>0$,

$$
\left|\left\{x \in \mathbb{R}^{n}: M_{\beta}(f)(x)>\lambda\right\}\right| \leq\left(\frac{C}{\lambda}\|f\|_{L^{1}}\right)^{\frac{n}{n-\beta}} .
$$

However the constant $C$, the bound of operator $M_{\beta}$, were not given explicit expression of depending on the parameters $p, q$ and $\beta$. In this paper, the bounds of the operator $\mathbb{H}_{\beta}$ from $L^{p}$ to $L^{q}$ and from $L^{1}$ to $L^{\frac{n}{n-\beta}, \infty}$ are explicitly worked out. Furthermore, we will show that the constant 1 is the bound of operator $\mathbb{H}_{\beta}$ from $L^{1}$ to $L^{\frac{n}{n-\beta}, \infty}$ and is beat possible, that is,

$$
\left\|\mathbb{H}_{\beta}\right\|_{L^{1} \rightarrow L^{\frac{n}{n-\beta}, \infty}}=1
$$

Throughout the paper, we use the following notation. The set $B(0,|x|)$ denotes a ball with center at the original point and radius $|x|$, and $|B(0,|x|)|$ denotes the volume of the ball $B(0,|x|) ;(B(0,|x|))^{c}=\mathbb{R}^{n} \backslash B(0,|x|)$.

\section{Sharp bounds for the Hardy type operator on product space}

Theorem 2.1 Let $1<p<\infty, m \in \mathbb{N}, n_{i} \in \mathbb{N}, x_{i} \in \mathbb{R}^{n_{i}}, i=1, \ldots$, m. If $f \in L^{p}\left(\mathbb{R}^{n_{1}} \times \mathbb{R}^{n_{2}} \times\right.$ $\left.\cdots \times \mathbb{R}^{n_{m}},|x|^{\vec{\alpha}}\right)$, where $|x|^{\vec{\alpha}}:=\left|x_{1}\right|^{\alpha_{1}}\left|x_{2}\right|^{\alpha_{2}} \cdots\left|x_{m}\right|^{\alpha_{m}}$ and $\alpha_{j}<(p-1) n_{j}$, then the Hardy type operator $\mathcal{H}_{m}$ defined in (3) is bounded on $L^{p}\left(\mathbb{R}^{n_{1}} \times \mathbb{R}^{n_{2}} \times \cdots \times \mathbb{R}^{n_{m}},|x|^{\vec{\alpha}}\right)$, moreover, the norm of $\mathcal{H}_{m}$ can be obtained as follows:

$$
\left\|\mathcal{H}_{m}\right\|_{L^{p}\left(|x|^{\vec{\alpha}}\right) \rightarrow L^{p}\left(|x|^{\vec{\alpha}}\right)}=\prod_{j=1}^{m}\left(\frac{p}{p-1-\frac{\alpha_{j}}{n_{j}}}\right) .
$$

Proof of Theorem 2.1 We merely give the proof with the case $m=2$ for the sake of clarity in writing, and the same is true for the general case $m>2$. We adapt some ideas and methods used in [12].

Set

$$
g_{f}\left(x_{1}, x_{2}\right)=\frac{1}{\omega_{n_{1}}} \frac{1}{\omega_{n_{2}}} \int_{S^{n_{1}-1}} \int_{S^{n_{2}-1}} f\left(\left|x_{1}\right| \xi_{1},\left|x_{2}\right| \xi_{2}\right) d \sigma\left(\xi_{1}\right) d \sigma\left(\xi_{2}\right),
$$

where $\omega_{n_{i}}=\frac{2 \pi \frac{n_{i}}{2}}{\Gamma\left(\frac{n_{i}}{2}\right)}$ and $x_{i} \in \mathbb{R}^{n_{i}}, i=1,2$. Obviously, $g$ is a nonnegative radial function with respect to the variables $x_{1}$ and $x_{2}$, respectively. In the following, we briefly call this function is a radial function on product space.

It follows that $\mathcal{H}_{2}\left(g_{f}\right)\left(x_{1}, x_{2}\right)$ is equal to

$$
\begin{aligned}
& \frac{1}{\left|B\left(0,\left|x_{1}\right|\right)\right|} \frac{1}{\left|B\left(0,\left|x_{2}\right|\right)\right|} \int_{B\left(0,\left|x_{1}\right|\right)} \int_{B\left(0,\left|x_{2}\right|\right)} \frac{1}{\omega_{n_{1}} \omega_{n_{2}}} \\
& \quad \times \int_{S^{n_{1}-1}} \int_{S^{n_{2}-1}} f\left(\left|y_{1}\right| \xi_{1},\left|y_{2}\right| \xi_{2}\right) d \sigma\left(\xi_{1}\right) d \sigma\left(\xi_{2}\right) d y_{1} d y_{2} \\
& =\frac{1}{\omega_{n_{1}} \omega_{n_{2}}} \int_{S^{n_{1}-1}} \int_{S^{n_{2}-1}} \frac{1}{\left|B\left(0,\left|x_{1}\right|\right)\right|} \frac{1}{\left|B\left(0,\left|x_{2}\right|\right)\right|}
\end{aligned}
$$




$$
\begin{aligned}
& \times \int_{B\left(0,\left|x_{1}\right|\right)} \int_{B\left(0,\left|x_{2}\right|\right)} f\left(\left|y_{1}\right| \xi_{1},\left|y_{2}\right| \xi_{2}\right) d y_{1} d y_{2} d \sigma\left(\xi_{1}\right) d \sigma\left(\xi_{2}\right) \\
= & \int_{S^{n_{1}-1}} \int_{S^{n_{2}-1}} \frac{1}{\left|B\left(0,\left|x_{1}\right|\right)\right|} \frac{1}{\left|B\left(0,\left|x_{2}\right|\right)\right|} \\
& \times \int_{0}^{\left|x_{1}\right|} \int_{0}^{\left|x_{2}\right|} f\left(r_{1} \xi_{1}, r_{2} \xi_{2}\right) r_{1}^{n_{1}-1} r_{2}^{n_{2}-1} d r_{1} d r_{2} d \sigma\left(\xi_{1}\right) d \sigma\left(\xi_{2}\right) \\
= & \frac{1}{\left|B\left(0,\left|x_{1}\right|\right)\right|} \frac{1}{\left|B\left(0,\left|x_{2}\right|\right)\right|} \int_{B\left(0,\left|x_{1}\right|\right)} \int_{B\left(0,\left|x_{2}\right|\right)} f\left(y_{1}, y_{2}\right) d y_{1} d y_{2} \\
= & \mathcal{H}_{2}(f)\left(x_{1}, x_{2}\right) .
\end{aligned}
$$

Using the generalized Minkowski's inequality and Hölder's inequality, we conclude that $\left\|g_{f}\right\|_{L^{p}(|x| \vec{\alpha})}$ is equal to

$$
\begin{aligned}
& \left(\int_{\mathbb{R}^{n_{1}}} \int_{\mathbb{R}^{n_{2}}}\left(\frac{1}{\omega_{n_{1}} \omega_{n_{2}}}\right)^{p}\right. \\
& \left.\quad \times\left(\int_{S^{n_{1}-1}} \int_{S^{n_{2}-1}} f\left(\left|x_{1}\right| \xi_{1},\left|x_{2}\right| \xi_{2}\right) d \sigma\left(\xi_{1}\right) d \sigma\left(\xi_{2}\right)\right)^{p}|x|^{\vec{\alpha}} d x_{1} d x_{2}\right)^{\frac{1}{p}} \\
& \leq \frac{1}{\omega_{n_{1}} \omega_{n_{2}}} \int_{S^{n_{1}-1}} \int_{S^{n_{2}-1}} \\
& \quad \times\left(\int_{\mathbb{R}^{n_{1}}} \int_{\mathbb{R}^{n_{2}}}\left(f\left(\left|x_{1}\right| \xi_{1},\left|x_{2}\right| \xi_{2}\right)\right)^{p}\left|x_{1}\right|^{\alpha_{1}}\left|x_{2}\right|^{\alpha_{2}} d x_{1} d x_{2}\right)^{\frac{1}{p}} d \sigma\left(\xi_{1}\right) d \sigma\left(\xi_{2}\right) \\
& \leq\left(\frac{1}{\omega_{n_{1}} \omega_{n_{2}}} \int_{S^{n_{1}-1}} \int_{S^{n_{2}-1}} \int_{\mathbb{R}^{n_{1}}} \int_{\mathbb{R}^{n_{2}}}\left(f\left(\left|x_{1}\right| \xi_{1},\left|x_{2}\right| \xi_{2}\right)\right)^{p}\right. \\
& \left.\quad \times\left|x_{1}\right|^{\alpha_{1}}\left|x_{2}\right|^{\alpha_{2}} d x_{1} d x_{2} d \sigma\left(\xi_{1}\right) d \sigma\left(\xi_{2}\right)\right)^{\frac{1}{p}} \\
& =\left(\int_{S^{n_{1}-1}} \int_{S^{n_{2}-1}} \int_{0}^{\infty} \int_{0}^{\infty}\left(f\left(r_{1} \xi_{1}, r_{2} \xi_{2}\right)\right)^{p} r_{1}^{\alpha_{1}+n_{1}-1} r_{2}^{\alpha_{2}+n_{2}-1} d r_{1} d r_{2} d \sigma\left(\xi_{1}\right) d \sigma\left(\xi_{2}\right)\right)^{\frac{1}{p}} \\
& =\|f\|_{L^{p}\left(\left.|x|\right|^{\alpha}\right)}
\end{aligned}
$$

Thus, we conclude that the following inequality:

$$
\frac{\left\|\mathcal{H}_{2}(f)\right\|_{L^{p}\left(|x|^{\vec{\alpha}}\right)}}{\|f\|_{L^{p}\left(|x|^{\mid \alpha}\right)}} \leq \frac{\left\|\mathcal{H}_{2}\left(g_{f}\right)\right\|_{L^{p}(|x| \vec{\alpha})}}{\left\|g_{f}\right\|_{L^{p}\left(\left.|x|\right|^{\vec{\alpha}}\right)}}
$$

holds provided that $\|f\|_{L^{p}\left(|x|^{\vec{\alpha}}\right)} \neq 0$. In addition, clearly if $f$ is a nonnegative radial function, then we have $g_{f}=f$. This means that the norm of the operator $\mathcal{H}_{2}$ is equal to the norm that $\mathcal{H}_{2}$ restricts to the set of nonnegative radial functions. Consequently, without loss of generality, it suffices to fulfil the proof of the theorem by assuming that $f$ is a nonnegative radial function.

Substituting the variables $z_{1}=\frac{y_{1}}{\left|x_{1}\right|}$ and $z_{2}=\frac{y_{2}}{\left|x_{2}\right|}$, we have that $\left\|\mathcal{H}_{2}(f)\right\|_{L^{p}(|x| \vec{\alpha})}$ equals

$$
\begin{aligned}
& \left(\int_{\mathbb{R}^{n_{1}}} \int_{\mathbb{R}^{n_{2}}}\left|\mathcal{H}(f)\left(x_{1}, x_{2}\right)\right|^{p}\left|x_{1}\right|^{\alpha_{1}}\left|x_{2}\right|^{\alpha_{2}} d x_{1} d x_{2}\right)^{\frac{1}{p}} \\
& =\left(\int_{\mathbb{R}^{n_{1}}} \int_{\mathbb{R}^{n_{2}}}\left(\frac{1}{\left|B\left(0,\left|x_{1}\right|\right)\right|\left|B\left(0,\left|x_{2}\right|\right)\right|}\right)^{p}\right.
\end{aligned}
$$




$$
\begin{aligned}
& \left.\times\left(\int_{\left|y_{1}\right|<\left|x_{1}\right|} \int_{\left|y_{2}\right|<\left|x_{2}\right|} f\left(y_{1}, y_{2}\right) d y_{1} d y_{2}\right)^{p}\left|x_{1}\right|^{\alpha_{1}}\left|x_{2}\right|^{\alpha_{2}} d x_{1} d x_{2}\right)^{\frac{1}{p}} \\
= & \frac{1}{v_{n_{1}} v_{n_{2}}}\left(\int_{\mathbb{R}^{n_{1}}} \int_{\mathbb{R}^{n_{2}}}\left(\int_{\left|z_{1}\right|<1} \int_{\left|z_{2}\right|<1} f\left(z_{1}\left|x_{1}\right|, z_{2}\left|x_{2}\right|\right) d z_{1} d z_{2}\right)^{p}\left|x_{1}\right|^{\alpha_{1}}\left|x_{2}\right|^{\alpha_{2}} d x_{1} d x_{2}\right)^{\frac{1}{p}} .
\end{aligned}
$$

Using the generalized Minkowski's inequality again and noting that $f$ is a radial function with respect to the first variable and the second variable, respectively, we have that $\left\|\mathcal{H}_{2}(f)\right\|_{L^{p}(|x| \vec{\alpha})}$ is not greater than

$$
\begin{aligned}
& \frac{1}{v_{n_{1}} v_{n_{2}}} \int_{\left|z_{1}\right|<1} \int_{\left|z_{2}\right|<1}\left(\int_{\mathbb{R}^{n_{1}}} \int_{\mathbb{R}^{n_{2}}}\left(f\left(z_{1}\left|x_{1}\right|, z_{2}\left|x_{2}\right|\right)\right)^{p}\left|x_{1}\right|^{\alpha_{1}}\left|x_{2}\right|^{\alpha_{2}} d x_{1} d x_{2}\right)^{\frac{1}{p}} d z_{1} d z_{2} \\
& =\frac{1}{v_{n_{1}} v_{n_{2}}} \int_{\left|z_{1}\right|<1} \int_{\left|z_{2}\right|<1}\left(\int_{\mathbb{R}^{n_{1}}} \int_{\mathbb{R}^{n_{2}}}\left(f\left(x_{1}, x_{2}\right)\right)^{p}\right. \\
& \left.\quad \times\left(\frac{\left|x_{1}\right|}{\left|z_{1}\right|}\right)^{\alpha_{1}}\left(\frac{\left|x_{2}\right|}{\left|z_{2}\right|}\right)^{\alpha_{2}} d x_{1} d x_{2}\right)^{\frac{1}{p}}\left|z_{1}\right|^{-\frac{n_{1}}{p}}\left|z_{2}\right|^{-\frac{n_{2}}{p}} d z_{1} d z_{2} \\
& =\frac{1}{v_{n_{1}} v_{n_{2}}} \int_{\left|z_{1}\right|<1} \int_{\left|z_{2}\right|<1}\left|z_{1}\right|^{-\frac{n_{1}+\alpha_{1}}{p}}\left|z_{2}\right|^{-\frac{n_{2}+\alpha_{2}}{p}} d z_{1} d z_{2}\|f\|_{L^{p}\left(|x|^{\mid \vec{\alpha})}\right)} \\
& =\prod_{j=1}^{2}\left(\frac{p}{p-1-\alpha_{j} / n_{j}}\right)\|f\|_{L^{p}(|x| \vec{\alpha})},
\end{aligned}
$$

where $v_{n_{i}}=\frac{\pi^{n_{i} / 2}}{\Gamma\left(1+n_{i} / 2\right)}$ is the volume of the unit ball in $\mathbb{R}^{n_{i}}, i=1,2$.

Therefore, it implies that

$$
\left\|\mathcal{H}_{2}\right\|_{L^{p}\left(|x|^{\vec{\alpha}}\right) \rightarrow L^{p}\left(|x|^{\vec{\alpha}}\right)} \leq \prod_{j=1}^{2}\left(\frac{p}{p-1-\alpha_{j} / n_{j}}\right) .
$$

Next, we need to prove the converse inequality.

For the purpose of getting the sharp bound, we set

$$
0<\varepsilon<\min \left\{1, \frac{\left(p_{1}-1\right) n}{p_{2}}, \frac{\left(p_{2}-1\right) n}{p_{2}}\right\}
$$

and define

$$
f_{\varepsilon}\left(x_{1}, x_{2}\right)=\left|x_{1}\right|^{-\frac{n_{1}+\alpha_{1}}{p}+\varepsilon}\left|x_{2}\right|^{-\frac{n_{2}+\alpha_{2}}{p}+\varepsilon} \chi_{\left\{\left|x_{1}\right|<1,\left|x_{2}\right|<1\right\}}\left(x_{1}, x_{2}\right) .
$$

It follows from the elementary calculation that $\left\|f_{\varepsilon}\right\|_{L^{p}\left(|x|^{\vec{\alpha}}\right)}$ is

$$
\begin{aligned}
& \left(\int_{\left|x_{1}\right|<1} \int_{\left|x_{2}\right|<1}\left|x_{1}\right|^{\left(\varepsilon-\frac{n_{1}+\alpha_{1}}{p}\right) p}\left|x_{2}\right|^{\left(\varepsilon-\frac{n_{2}+\alpha_{2}}{p}\right) p}|x|^{\vec{\alpha}} d x_{2} d x_{1}\right)^{\frac{1}{p}} \\
& =\left(\int_{\left|x_{1}\right|<1}\left|x_{1}\right|^{\left(-\frac{n_{1}}{p}+\varepsilon\right) p} d x_{1}\right)^{1 / p}\left(\int_{\left|x_{2}\right|<1}\left|x_{2}\right|^{\left(-\frac{n_{2}}{p}+\varepsilon\right) p} d x_{2}\right)^{\frac{1}{p}} \\
& =\left(\frac{\omega_{n_{1}}}{p \varepsilon}\right)^{1 / p}\left(\frac{\omega_{n_{2}}}{p \varepsilon}\right)^{\frac{1}{p}} .
\end{aligned}
$$


We rewrite $\mathcal{H}_{2}\left(f_{\varepsilon}\right)$ as follows:

$$
\begin{aligned}
\mathcal{H}_{2}\left(f_{\varepsilon}\right)\left(x_{1}, x_{2}\right)= & \frac{1}{\left|B\left(0,\left|x_{1}\right|\right)\right|\left|B\left(0,\left|x_{2}\right|\right)\right|} \int_{\left|y_{1}\right|<\left|x_{1}\right|} \int_{\left|y_{2}\right|<\left|x_{2}\right|} f_{\varepsilon}\left(y_{1}, y_{2}\right) d y_{1} d y_{2} \\
= & \frac{1}{|B(0,1)||B(0,1)|} \int_{\left|z_{1}\right|<1} \int_{\left|z_{2}\right|<1} f_{\varepsilon}\left(z_{1}\left|x_{1}\right|, z_{2}\left|x_{2}\right|\right) d z_{1} d z_{2} \\
= & \frac{\left|x_{1}\right|^{-\frac{n_{1}+\alpha_{1}}{p}+\varepsilon}\left|x_{2}\right|^{-\frac{n_{2}+\alpha_{2}}{p}+\varepsilon}}{|B(0,1)||B(0,1)|} \\
& \times \int_{\left\{\left|z_{1}\right|<1,\left|z_{1}\right|<1 /\left|x_{1}\right|\right\}} \int_{\left\{\left|z_{2}\right|<1,\left|z_{2}\right|<1 /\left|x_{2}\right|\right\}}\left|z_{1}\right|^{-\frac{n_{1}+\alpha_{1}}{p}+\varepsilon}\left|z_{2}\right|^{-\frac{n_{2}+\alpha_{2}}{p}+\varepsilon} d z_{2} d z_{1} .
\end{aligned}
$$

Thus, we estimate the norm of $\left\|\mathcal{H}_{2}\left(f_{\varepsilon}\right)\right\|_{L^{p}}$ as follows:

$$
\begin{aligned}
\left\|\mathcal{H}_{2}\left(f_{\varepsilon}\right)\right\|_{L^{p}\left(|x|^{\vec{\alpha})}\right)=}^{p} & \left.\frac{1}{\left(v_{n_{1}} v_{n_{2}}\right)^{p}} \int_{\mathbb{R}^{n_{1}}} \int_{\mathbb{R}^{n_{2}}}\left|\int_{\left\{\left|z_{1}\right|<1,\left|z_{1}\right|<\frac{1}{\left.\mid x_{1}\right\}}\right.} \int_{\left\{\left|z_{2}\right|<1,\left|z_{2}\right|<\frac{1}{\left|x_{2}\right|}\right\}}\right| z_{1}\right|^{-\frac{n_{1}+\alpha_{1}}{p}+\varepsilon} \\
& \times\left.\left|z_{2}\right|^{-\frac{n_{2}+\alpha_{1}}{p}+\varepsilon} d z_{2} d z_{1}\right|^{p}\left|x_{1}\right|^{p \varepsilon-n_{1}-\alpha_{1}}\left|x_{2}\right|^{p \varepsilon-n_{2}-\alpha_{2}}|x|^{\vec{\alpha}} d x_{2} d x_{1} \\
\geq & \left.\frac{1}{\left(v_{n_{1}} v_{n_{2}}\right)^{p}} \int_{\left|x_{1}\right|<1} \int_{\left|x_{2}\right|<1}\left|\int_{\left|z_{1}\right|<1} \int_{\left|z_{2}\right|<1}\right| z_{1}\right|^{-\frac{n_{1}+\alpha_{1}}{p}+\varepsilon} \\
& \times\left.\left|z_{2}\right|^{-\frac{n_{2}+\alpha_{2}}{p}+\varepsilon} d z_{2} d z_{1}\right|^{p}\left|x_{1}\right|^{p \varepsilon-n_{1}}\left|x_{2}\right|^{p \varepsilon-n_{2}} d x_{2} d x_{1} \\
= & \frac{1}{\left(v_{n_{1}} v_{n_{2}}\right)^{p}} \frac{\omega_{n_{1}}}{p \varepsilon} \frac{\omega_{n_{2}}}{p \varepsilon}\left(\int_{\left|z_{1}\right|<1} \int_{\left|z_{2}\right|<1}\left|z_{1}\right|^{-\frac{n_{1}+\alpha_{1}}{p}+\varepsilon}\left|z_{2}\right|^{-\frac{n_{2}+\alpha_{2}}{p}+\varepsilon} d z_{2} d z_{1}\right)^{p} \\
= & \frac{1}{\left(v_{n_{1}} v_{n_{2}}\right)^{p}}\left(\frac{\omega_{n_{1}}}{n_{1}\left(1-1 / p-\alpha_{1} / p n_{1}+\varepsilon / n_{1}\right)}\right)^{p} \\
& \times\left(\frac{\omega_{n_{2}}}{n_{2}\left(1-1 / p-\alpha_{2} / p n_{2}+\varepsilon / n_{2}\right)}\right)^{p}\left\|f_{\varepsilon}\right\|_{L^{p}}^{p} \\
= & \left(\frac{p}{p-1-\alpha_{1} / n+p \varepsilon / n_{1}} \frac{p}{p-1-\alpha_{2} / n+p \varepsilon / n_{2}}\right)^{p}\left\|f_{\varepsilon}\right\|_{L^{p}\left(|x|^{\vec{\alpha}}\right)^{p}}^{p}
\end{aligned}
$$

Therefore, it implies that

$$
\frac{\left\|\mathcal{H}_{2}\left(f_{\varepsilon}\right)\right\|_{L^{p}\left(|x|^{\vec{\alpha}}\right)}}{\left\|f_{\varepsilon}\right\|_{L^{p}\left(\left.|x|\right|^{\vec{\alpha}}\right)}} \geq \frac{p}{p-1-\alpha_{1} / n+p \varepsilon / n_{1}} \cdot \frac{p}{p-1-\alpha_{2} / n+p \varepsilon / n_{2}} .
$$

Consequently, using the definition of the norm of the operator and letting $\varepsilon \rightarrow 0$, we conclude that

$$
\left\|\mathcal{H}_{2}\right\|_{L^{p}\left(|x|^{\vec{\alpha}}\right) \rightarrow L^{p}(|x| \vec{\alpha})} \geq \prod_{j=1}^{2}\left(\frac{p}{p-1-\alpha_{j} / n_{j}}\right) .
$$

This finishes the proof of the theorem.

\section{Explicit bounds for the fractional Hardy operator}

Theorem 3.1 Suppose that $0 \leq \beta<n, 1<p \leq \frac{n}{\beta}$ and $\frac{1}{p}-\frac{1}{q}=\frac{\beta}{n}$.

(i) Iff $\in L^{p}\left(\mathbb{R}^{n}\right)$, then we have

$$
\left\|\mathbb{H}_{\beta}(f)\right\|_{L^{q}} \leq C\|f\|_{L^{p}}
$$


where

$$
\left(\frac{p}{q}\right)^{1 / q}\left(\frac{p}{p-1}\right)^{1 / q}\left(\frac{q}{q-1}\right)^{1-1 / q}\left(1-\frac{p}{q}\right)^{1 / p-1 / q} \leq C \leq\left(\frac{p}{p-1}\right)^{\frac{p}{q}} .
$$

(ii) If $f \in L^{1}\left(\mathbb{R}^{n}\right)$, then for any $\lambda>0$,

$$
\left|\left\{x \in \mathbb{R}^{n}:\left|\mathbb{H}_{\beta}(f)(x)\right|>\lambda\right\}\right| \leq\left(\frac{1}{\lambda}\|f\|_{L^{1}}\right)^{\frac{n}{n-\beta}} .
$$

Moreover,

$$
\left\|\mathbb{H}_{\beta}\right\|_{L^{1} \rightarrow L^{\frac{n}{n-\beta}}, \infty}=1 .
$$

Proof of (i) of Theorem 3.1 Set $\omega_{n}=2 \pi^{\frac{n}{2}} / \Gamma\left(\frac{n}{2}\right)$ as above. Let

$$
g_{f}(y)=\frac{1}{\omega_{n}} \int_{|\xi|=1} f(|y| \xi) d \xi, \quad y \in \mathbb{R}^{n}
$$

Clearly, $g_{f}$ is a radial function.

$$
\begin{aligned}
\mathbb{H}_{\beta}\left(g_{f}\right)(x) & =\frac{1}{\left(v_{n}|x|^{n}\right)^{1-\frac{\beta}{n}}} \int_{|y|<|x|} g_{f}(y) d y \\
& =\frac{1}{\left(v_{n}|x|^{n}\right)^{1-\frac{\beta}{n}}} \int_{|y|<|x|}\left(\frac{1}{\omega_{n}} \int_{|\xi|=1} f(|y| \xi) d \xi\right) d y \\
& =\frac{1}{\left(v_{n}|x|^{n}\right)^{1-\frac{\beta}{n}}} \int_{|\xi|=1}\left(\frac{1}{\omega_{n}} \int_{|y|<|x|} f(|y| \xi) d y\right) d \xi \\
& =\frac{1}{\left(v_{n}|x|^{n}\right)^{1-\frac{\beta}{n}}} \int_{|y|<|x|} f(y) d y \\
& =\mathbb{H}_{\beta}(f)(x) .
\end{aligned}
$$

Using the generalized Minkowski inequality and Hölder's inequality, we have that

$$
\begin{aligned}
\left\|g_{f}\right\|_{L^{p}} & =\left(\int_{\mathbb{R}^{n}}\left|g_{f}(x)\right|^{p} d x\right)^{\frac{1}{p}} \\
& \leq \frac{1}{\omega_{n}} \int_{|\xi|=1}\left(\int_{\mathbb{R}^{n}}|f(|x| \xi)|^{p} d x\right)^{\frac{1}{p}} d \xi \\
& =\frac{\omega_{n}^{1 / p}}{\omega_{n}} \int_{|\xi|=1}\left(\int_{0}^{\infty}|f(t \xi)|^{p} t^{n-1} d t\right)^{1 / p} d \xi \\
& \leq \frac{\omega_{n}^{1 / p}}{\omega_{n}}\left(\int_{|\xi|=1} \int_{0}^{\infty}|f(t \xi)|^{p} t^{n-1} d t d \xi\right)^{1 / p}\left(\int_{|\xi|=1} d \xi\right)^{1-1 / p} \\
& =\|f\|_{L^{p}} .
\end{aligned}
$$

Therefore, we have that

$$
\frac{\left\|\mathbb{H}_{\beta}(f)\right\|_{L^{q}}}{\|f\|_{L^{p}}} \leq \frac{\left\|\mathbb{H}_{\beta}\left(g_{f}\right)\right\|_{L^{q}}}{\left\|g_{f}\right\|_{L^{p}}}
$$


As above, this means that the norm of the operator $\mathbb{H}_{\beta}$ from $L^{p}$ to $L^{q}$ is equal to the norm that $\mathbb{H}_{\beta}$ restricts to radial functions. Consequently, without loss of generality, it suffices to carry out the proof of the theorem by assuming that $f$ is a radial function.

A simple estimate implies that $\left\|\mathbb{H}_{\beta}(f)\right\|_{L^{q}}$ equals

$$
\begin{aligned}
& \left(\int_{\mathbb{R}^{n}}\left|\frac{1}{\left(v_{n}|x|^{n}\right)^{1-\frac{\beta}{n}}} \int_{|y|<|x|} f(y) d y\right|^{q} d x\right)^{\frac{1}{q}} \\
& \quad=v_{n}^{\frac{\beta}{n}-1+\frac{p}{q}}\left(\int_{\mathbb{R}^{n}}\left|\int_{|y|<|x|} f(y) d y\right|^{q-p}|\mathcal{H}(f)(x)|^{p}|x|^{n q\left(\frac{\beta}{n}-1\right)+p n} d x\right)^{\frac{1}{q}} \\
& \leq v_{n}^{\frac{\beta}{n}-1+\frac{p}{q}}\|f\|_{p}^{\frac{q-p}{q}}\left(\int_{\mathbb{R}^{n}}\left(v_{n}|x|^{n}\right)^{\left(1-\frac{1}{p}\right)(q-p)}|\mathcal{H}(f)(x)|^{p}|x|^{n q\left(\frac{\beta}{n}-1\right)+p n} d x\right)^{\frac{1}{q}} \\
& \quad=\|f\|_{p}^{\frac{q-p}{q}}\left(\int_{\mathbb{R}^{n}}|\mathcal{H}(f)(x)|^{p} d x\right)^{\frac{1}{q}} \leq\left(\frac{p}{p-1}\right)^{\frac{p}{q}}\|f\|_{L^{p}},
\end{aligned}
$$

where we use the well-known consequence that $\mathcal{H}$ is bounded on $L^{p}\left(\mathbb{R}^{n}\right)$ with the sharp bound $\frac{p}{p-1}$ and the following relationship:

$$
(q-p)\left(1-\frac{1}{p}\right)+p+\left(\frac{\beta}{n}-1\right) q=0
$$

To obtain a better lower bound of $\|\mathcal{H}\|_{L^{p} \rightarrow L^{q}}$, we can take $f_{0}(x)=|x|^{\frac{n C_{1}}{p}} \chi_{\{|x|<1\}}(x), C_{1}>-1$. Then

$$
\left\|f_{0}\right\|_{L^{p}}^{p}=\frac{v_{n}}{C_{1}+1} .
$$

We have

$$
\begin{aligned}
& \left\|\mathbb{H}_{\beta}\left(f_{0}\right)\right\|_{L^{q}}^{q}=\int_{|x|>1}\left(\frac{1}{\left(v_{n}|x|^{n}\right)^{(1-\beta / n)}} \int_{|y|<1}|y|^{\frac{n C_{1}}{p}} d y\right)^{q} d x \\
& +\int_{|x| \leq 1}\left(\frac{1}{\left(v_{n}|x|^{n}\right)^{(1-\beta / n)}} \int_{|y|<|x|}|y|^{\frac{n C_{1}}{p}} d y\right)^{q} d x \\
& =\frac{\omega_{n}^{q}}{v_{n}^{\left(1-\frac{\beta}{n}\right) q}\left(n C_{1} / p+n\right)^{q}}\left(\int_{|x|>1}|x|^{(\beta-n) q} d x+\int_{|x| \leq 1}|x|^{\left(\beta+\frac{n C_{1}}{p}\right) q} d x\right) \\
& =\frac{\omega_{n}^{(1+q)}}{v_{n}^{\left(1-\frac{\beta}{n}\right) q}\left(n C_{1} / p+n\right)^{q}} \frac{p}{q n}\left(\frac{1}{1+C_{1}}+\frac{1}{p-1}\right) \text {. }
\end{aligned}
$$

So, we have

$$
\begin{aligned}
\frac{\left\|\mathbb{H}_{\beta}\left(f_{0}\right)\right\|_{L^{q}}}{\left\|f_{0}\right\|_{L^{p}}} & =\left(\frac{\omega_{n}^{(1+q)}}{v_{n}^{(1-\beta / n) q}\left(n C_{1} / p+n\right)^{q}} \frac{p}{q n}\left(\frac{1}{1+C_{1}}+\frac{1}{p-1}\right)\right)^{\frac{1}{q}}\left(\frac{C_{1}+1}{v_{n}}\right)^{\frac{1}{p}} \\
& =\left(\frac{p}{q}\right)^{1 / q} \frac{\left(1+C_{1}\right)^{1 / p}}{1+C_{1} / p}\left(\frac{1}{1+C_{1}}+\frac{1}{p-1}\right)^{\frac{1}{q}} \\
& =\left(\frac{p}{q}\right)^{1 / q}\left(\frac{p}{p-1}\right)^{1 / q} \frac{\left(1+C_{1}\right)^{\frac{1}{p}-\frac{1}{q}}}{\left(1+C_{1} / p\right)^{1-\frac{1}{q}}}
\end{aligned}
$$


Let $g(t)=(1+t)^{\frac{1}{p}-\frac{1}{q}}(1+t / p)^{-\left(1-\frac{1}{q}\right)}, t>-1$. Taking $g^{\prime}\left(t^{*}\right)=0$, we get $t^{*}=-p / q$, where $g^{\prime}$ is the derivative of the function $g$. It can be easily verified that the function $g(t)$ defined over the open interval $(-1, \infty)$ has a unique global maximum at the point $t^{*}$. Taking $C_{1}=-p / q$ together with inequality (5), we get

$$
\left\|\mathbb{H}_{\beta}\right\|_{L^{p} \rightarrow L^{q}} \geq\left(\frac{p}{q}\right)^{1 / q}\left(\frac{p}{p-1}\right)^{1 / q}\left(\frac{q}{q-1}\right)^{1-1 / q}\left(1-\frac{p}{q}\right)^{1 / p-1 / q} .
$$

Proof of (ii) of Theorem 3.1 Since

$$
\mathbb{H}_{\beta}(f)(x)=\frac{1}{\left(v_{n}|x|^{n}\right)^{1-\frac{\beta}{n}}} \int_{|y|<|x|}|f(y)| d y \leq \frac{1}{\left(v_{n}|x|^{n}\right)^{1-\frac{\beta}{n}}}\|f\|_{L^{1}},
$$

we have that

$$
\begin{aligned}
\left|\left\{x \in \mathbb{R}^{n}:\left|\mathbb{H}_{\beta}(f)(x)\right|>\lambda\right\}\right| & \leq\left|\left\{x \in \mathbb{R}^{n}: \frac{1}{\left(v_{n}|x|^{n}\right)^{1-\frac{\beta}{n}}}\|f\|_{L^{1}}>\lambda\right\}\right| \\
& =\left|\left\{x \in \mathbb{R}^{n}:|x|<\left(\frac{\|f\|_{L^{1}}}{\lambda v_{n}^{1-\frac{\beta}{n}}}\right)^{\frac{1}{n-\beta}}\right\}\right| \\
& =\left(\frac{\|f\|_{L^{1}}}{\lambda}\right)^{\frac{n}{n-\beta}} .
\end{aligned}
$$

Next, we will show that the constant 1 is a sharp bound by constructing a suitable function. In fact, set

$$
g(x)=\chi_{|x|<1}(x)
$$

we have

$$
\|g\|_{L^{1}}=v_{n}
$$

It follows that

$$
\mathbb{H}_{\beta}(g)(x)=\frac{1}{\left(v_{n}|x|^{n}\right)^{1-\frac{\beta}{n}}} \int_{|y|<|x|} \chi_{|y|<1}(y) d y=\frac{|\{y:|y|<\min \{|x|, 1\}\}|}{\left(v_{n}|x|^{n}\right)^{1-\frac{\beta}{n}}} .
$$

We assert that $\mathbb{H}_{\beta}(g)(x) \leq v_{n}^{\frac{\beta}{n}}$ for all $x \in \mathbb{R}^{n}$. We rewrite

$$
\left\{x \in \mathbb{R}^{n}: \mathbb{H}_{\beta}(g)(x)>v_{n}^{\frac{\beta}{n}}\right\}:=A \cup B
$$

where

$$
\begin{aligned}
& A:=B(0,1) \cap\left\{x \in \mathbb{R}^{n}: \mathbb{H}_{\beta}(g)(x)>v_{n}^{\frac{\beta}{n}}\right\}, \\
& B:=(B(0,1))^{c} \cap\left\{x \in \mathbb{R}^{n}: \mathbb{H}_{\beta}(g)(x)>v_{n}^{\frac{\beta}{n}}\right\} .
\end{aligned}
$$

Next, we estimate two sets $A$ and $B$, respectively. 
If $x \in A$, then $|x|<1$. It follows that

$$
\mathbb{H}_{\beta}(g)(x) \leq \frac{|\{y:|y|<|x|\}|}{\left(v_{n}|x|^{n}\right)^{1-\frac{\beta}{n}}}=\left(v_{n}|x|^{n}\right)^{\frac{\beta}{n}}<v_{n}^{\frac{\beta}{n}} .
$$

If $x \in B$, then $|x| \geq 1$. It follows that

$$
\mathbb{H}_{\beta}(g)(x) \leq \frac{|\{y:|y|<1\}|}{\left(v_{n}|x|^{n}\right)^{1-\frac{\beta}{n}}} \leq v_{n}^{\frac{\beta}{n}}
$$

Consequently, we have $A=B=\emptyset$. This implies that $\mathbb{H}_{\beta}(g)(x) \leq v_{n}^{\frac{\beta}{n}}$.

For any $0<\lambda<v_{n}^{\frac{\beta}{n}}$, we conclude that

$$
\begin{aligned}
\mid\{x & \left.\in \mathbb{R}^{n}:\left|\mathbb{H}_{\beta}(g)(x)\right|>\lambda\right\} \mid \\
& =\left|B(0,1) \cap\left\{x \in \mathbb{R}^{n}: v_{n}^{\frac{\beta}{n}}|x|^{\beta}>\lambda\right\}\right|+\left|(B(0,1))^{c} \cap\left\{x \in \mathbb{R}^{n}: \frac{v_{n}^{\frac{\beta}{n}}}{|x|^{n-\beta}}>\lambda\right\}\right| \\
& =\left|\left\{x \in \mathbb{R}^{n}: \frac{\lambda^{1 / \beta}}{v_{n}^{1 / n}}<|x|<1\right\}\right|+\left|\left\{x \in \mathbb{R}^{n}: 1 \leq|x|<\left(\frac{v_{n}^{\frac{\beta}{n}}}{\lambda}\right)^{\frac{1}{n-\beta}}\right\}\right| \\
& =\left(\frac{v_{n}}{\lambda}\right)^{\frac{n}{n-\beta}}-\lambda^{\frac{n}{\beta}} .
\end{aligned}
$$

If there exists a constant $C$ such that

$$
\left|\left\{x \in \mathbb{R}^{n}:\left|\mathbb{H}_{\beta}(f)(x)\right|>\lambda\right\}\right| \leq\left(\frac{C\|f\|_{L^{1}}}{\lambda}\right)^{\frac{n}{n-\beta}}
$$

holds for all $f \in L^{1}\left(\mathbb{R}^{n}\right)$. Then we can choose that

$$
f(x)=\chi_{|x|<1}(x)
$$

It follows from equality (7) that

$$
\left(\frac{v_{n}}{\lambda}\right)^{\frac{n}{n-\beta}}-\lambda^{\frac{n}{\beta}} \leq\left(\frac{C v_{n}}{\lambda}\right)^{\frac{n}{n-\beta}}
$$

always holds for every $0<\lambda<v_{n}^{\frac{\beta}{n}}$. Letting $\lambda \rightarrow 0^{+}$, this forces that $C \geq 1$. This means that the constant 1 is sharp.

At the end of this paper, we revisit the lower bound of $\left\|\mathbb{H}_{\beta}\right\|_{L^{p} \rightarrow L^{q}}$. Using L'Hospital's rule, we obtain that

$$
\lim _{q \rightarrow p^{+}}\left(1-\frac{p}{q}\right)^{1 / p-1 / q}=1 .
$$

This implies that

$$
\|\mathcal{H}\|_{L^{p} \rightarrow L^{p}}=\frac{p}{p-1} .
$$


Remark 3.1 If $\beta=0$, the operator $\mathbb{H}_{\beta}$ is reduced to the classical Hardy operator $\mathcal{H}$. In addition, in order to study the endpoint estimate for Hardy operator, we in [13] modified the definition of (2) as follows:

$$
\tilde{\mathcal{H}}(f)(x)=\frac{1}{|B(0,|x|)|} \int_{|y|<|x|} f(y) d y, \quad x \in \mathbb{R}^{n} \backslash\{0\},
$$

where $f$ is a measurable function on $\mathbb{R}^{n}$. In fact, the operators $\tilde{\mathcal{H}}$ and $\mathcal{H}$ enjoy the same boundedness property for $L^{p} \rightarrow L^{p}$ and $L^{1} \rightarrow L^{1, \infty}$, but they do not have the same property involving the Hardy space $H^{1}$. For instance, the operator $\tilde{\mathcal{H}}$ is bounded from $H^{1}$ to $L^{1}$; but the operator $\mathcal{H}$ is not since $\mathcal{H}$ is nonnegative (cf. [13]).

The well-known fact is that $\|\mathcal{H}\|_{L^{p} \rightarrow L^{p}}=\frac{p}{p-1}$ (cf. [2]) and $\|\tilde{\mathcal{H}}\|_{L^{1} \rightarrow L^{1, \infty}}=\|\mathcal{H}\|_{L^{1} \rightarrow L^{1, \infty}}=1$ (cf. [13]). On account of these facts, we guess that the lower bound of (i) in Theorem 3.1 is sharp.

\section{Competing interests}

The authors declare that they have no competing interests.

\section{Authors' contributions}

All authors contributed equally and significantly in writing this paper. All authors read and approved the final manuscript.

\section{Author details}

'School of Mathematical Sciences, Beijing Normal University, Beijing, 100875, P.R. China. ${ }^{2}$ School of Mathematics Science, University of Chinese Academy of Sciences, Beijing, 100190, P.R. China. ${ }^{3}$ Department of Mathematics, Shanghai University, Shanghai, 200444, P.R. China.

\section{Acknowledgements}

The authors would like to express their thanks to the referees for valuable advice regarding a previous version of this paper. This research was supported by NNSF of China (Grant Nos. 11271175, 10931001, 10771221, 11271162 and 11201287), NSF of Beijing (Grant No. 1092004) and NSF of Zhejiang (Grant No. Y6110074). This work was also supported by the FCDU in Shanghai and the KLMCS (Beijing Normal University), Ministry of Education, China.

\section{Received: 11 July 2012 Accepted: 21 March 2013 Published: 3 April 2013}

\section{References}

1. Hardy, GH: Note on a theorem of Hilbert. Math. Z. 6, 314-317 (1920)

2. Christ, M, Grafakos, L: Best constants for two nonconvolution inequalities. Proc. Am. Math. Soc. 123, 1687-1693 (1995)

3. Bényi, Á, Oh, T: Best constants for certain multilinear integral operators. J. Inequal. Appl. 2006, Article ID 28582 (2006)

4. Wang, SM, Lu, SZ, Yan, DY: Explicit constants for Hardy's inequality with power weight on $n$-dimensional product spaces. Sci. China Math. 55, 2469-2480 (2012)

5. Muckenhoupt, B: Weighted norm inequalities for classical operators. Proc. Symp. Pure Math. 35, 69-83 (1979)

6. Pachpatte, BG: On multivariate Hardy type inequalities. An. ştiinț. Univ. "Al... Cuza" laşi, Mat. 38, 355-361 (1992)

7. Kufner, A, Maligranda, L, Persson, LE: The Hardy Inequality. About Its History and Some Related Results. Vydavatelsky Servis Publishing House, Pilsen (2007)

8. Kufner, A, Persson, LE: Weighted Inequalities of Hardy Type. World Scientific, Singapore (2003)

9. Hanjs, Z, Pearce, CEM, Pecaric, J: Multivariate Hardy-type inequalities. Tamkang J. Math. 2(31), 149-158 (2000)

10. Fu, ZW, Liu, ZG, Lu, SZ, Wang, HB: Characterization for commutators of $n$-dimensional fractional Hardy operators. Sci. China Ser. A 50, 1418-1426 (2007)

11. Lu, SZ, Ding, Y, Yan, DY: Singular Integral and Related Topics. World Scientific, Singapore (2007)

12. Fu, ZW, Grafakos, L, Lu, SZ, Zhao, FY: Sharp bounds for m-linear Hardy and Hilbert operators. Houst. J. Math. 38, 225-244 (2012)

13. Zhao, FY, Fu, ZW, Lu, SZ: Endpoint estimates for $n$-dimensional Hardy operators and their commutators. Sci. China Math. 55, 1977-1990 (2012)

doi:10.1186/1029-242X-2013-148

Cite this article as: Lu et al.: Sharp bounds for Hardy type operators on higher-dimensional product spaces. Journal of Inequalities and Applications 2013 2013:148. 